\title{
Studies of Asymmetries in Semileptonic B decays at $\mathrm{LHCb}$
}

Thomas RUF ${ }^{* \dagger}$

CERN

E-mail: Thomas.Rufecern.ch

$\mathrm{CP}$ violating and $\mathrm{CP}$ conserving asymmetries in semileptonic $B$ decays are studied with the LHCb detector using a data sample corresponding to an integrated luminosity of $1 \mathrm{fb}^{-1}$ of $p p$ collisions at $\sqrt{s}=7 \mathrm{TeV}$, collected during the 2011 run of the LHC. The CP-violating, flavour specific asymmetry, $A_{f s}^{s}$ is measured to be $\left(-0.06 \pm 0.50_{\text {stat. }} \pm 0.36_{\text {sys. }}\right) \%$, which is the most precise measurement of this quantity to date and agrees with the Standard Model prediction. The $B^{0}$ and $B_{s}^{0}$ mixing frequencies are measured with high precision for the first time from semileptonic decays only and are found to be $\Delta m_{d}=\left(0.503 \pm 0.011_{\text {stat. }} \pm 0.013_{\text {sys. }}\right) \mathrm{ps}^{-1}$ and $\Delta m_{s}=\left(17.93 \pm 0.22_{\text {stat. }} \pm 0.15_{\text {sys. }}\right) \mathrm{ps}^{-1}$, respectively.

The European Physical Society Conference on High Energy Physics -EPS-HEP2013

18-24 July 2013

Stockholm, Sweden

* Speaker.

†n behalf of the LHCb collaboration 


\section{Introduction}

The $\mathrm{LHCb}$ detector is a single-arm magnetic dipole spectrometer designed for high precision measurements of $\mathrm{CP}$ violation and rare decays in the beauty and charm sector. It therefore has excellent tracking and vertexing capabilities. The Vertex Locator (VELO) consists of silicon microstrip modules, distributed in 23 stations arranged along the beam direction, providing an impact parameter resolution for high- $\mathrm{P}_{\mathrm{T}}$ particles down to $15 \mu \mathrm{m}$. Particles traversing from the interaction region to the downstream tracking stations experience a bending-field integral of around $4 \mathrm{Tm}$. The other unique feature of the experiment is two Rich detectors which provide hadron identification over a large momentum range. The Muon and Calorimeter systems are read-out at $40 \mathrm{MHz}$ and the measured transverse momentum of muons and energy of hadrons and photons are input to the first-level trigger. The high level trigger receives events at a rate of $1 \mathrm{MHz}$ and runs essentially the full offline reconstruction tuned to the trigger time constraints on about 29000 logical CPU cores in parallel. The polarity of the dipole magnet is reversed every $\approx 100 \mathrm{pb}^{-1}$ of integrated luminosity to minimize detector induced charge asymmetries. More details about the detector and trigger can be found elsewhere $[1,2]$. The talk summarizes two LHCb analyses $[3,4]$ using data collected in 2011 corresponding to an integrated luminosity of $1 \mathrm{fb}^{-1}$ of $p p$ collisions at $\sqrt{s}=7 \mathrm{TeV}$.

\section{Neutral Meson Mixing and CP violation}

The time evolution of flavour in a neutral meson state is governed by an effective Schrödinger equation:

$$
i \frac{\partial}{\partial t}\left(\begin{array}{l}
B(t) \\
\bar{B}(t)
\end{array}\right)=\Lambda\left(\begin{array}{c}
B(t) \\
\bar{B}(t)
\end{array}\right) .
$$

As result of using the Wigner-Weisskopf approach making use of the fact that the weak interaction responsible for flavour changing processes is much weaker than the strong interaction, the $2 \times 2$ complex matrix $\Lambda$ can be divided into a hermitian part, the mass matrix, and an antihermitian part, the decay matrix:

$$
\Lambda=\left(\begin{array}{cc}
M_{11} & M_{12} \mathrm{e}^{\mathrm{i} \varphi_{\mathrm{M}}} \\
M_{12} \mathrm{e}^{-\mathrm{i} \varphi_{\mathrm{M}}} & M_{22}
\end{array}\right)-\frac{i}{2}\left(\begin{array}{cc}
\Gamma_{11} & \Gamma_{12} \mathrm{e}^{\mathrm{i} \varphi_{\Gamma}} \\
\Gamma_{12} \mathrm{e}^{-\mathrm{i} \varphi_{\Gamma}} & \Gamma_{22}
\end{array}\right) .
$$

The eigenstates of $\Lambda$ are called $B_{H}$ and $B_{L}$. In the limit of $\Gamma_{12} / M_{12} \ll 1$ their mass and lifetime difference can be expressed as $\Delta m=m_{H}-m_{L}=2 M_{12}$ and $\Delta \Gamma=\Gamma_{H}-\Gamma_{L}=2 \Gamma_{12} \cos \left(\varphi_{\Gamma}-\varphi_{M}\right)$ respectively. The time evolution of flavour is then given by:

$$
\begin{aligned}
& R\left(\begin{array}{c}
B \rightarrow B \\
\bar{B} \rightarrow \bar{B}
\end{array}\right)=\frac{1}{2} \mathrm{e}^{-\mathrm{i} \bar{\Gamma}}\left(\cosh \frac{\Delta \Gamma t}{2}+\cos \Delta m t\right) \\
& R\left(\begin{array}{c}
B \rightarrow \bar{B} \\
\bar{B} \rightarrow B
\end{array}\right)=\frac{2}{|\Delta \Lambda|^{2}} \mathrm{e}^{-\mathrm{i} \bar{\Gamma}}\left(\cosh \frac{\Delta \Gamma t}{2}-\cos \Delta m t\right)\left(\begin{array}{l}
\left|\Lambda_{12}\right|^{2} \\
\left|\Lambda_{21}\right|^{2}
\end{array}\right)
\end{aligned}
$$

with $\Delta \Lambda$ the difference of the two eigenvalues of $\Lambda$ and $\bar{\Gamma}$ the average lifetime of the eigenstates. The asymmetry between the two flavour changing rates is a direct manifestation of $T$ non-invariance of 
the weak Hamiltonian without any assumptions on $C P T$. This asymmetry is called flavour specific asymmetry and given by:

$$
A_{f s}=\frac{R(B \rightarrow \bar{B})-R(\bar{B} \rightarrow B)}{R(B \rightarrow \bar{B})+R(\bar{B} \rightarrow B)}=\frac{\left|\Lambda_{12}\right|^{2}-\left|\Lambda_{21}\right|^{2}}{\left|\Lambda_{12}\right|^{2}+\left|\Lambda_{21}\right|^{2}}=\frac{\Gamma_{12}}{M_{12}} \sin \left(\varphi_{\Gamma}-\varphi_{M}\right)
$$

In the kaon system, this asymmetry is historically referenced to as the "Kabir Asymmetry"[5] or $A_{T}$ and had been measured for the first time by CPLEAR in 1998, $A_{T}^{\text {kaon }}=(6.6 \pm 1.3 \pm 1.0) \times 10^{-3}[6]$. In the $B$ systems, this asymmetry can be calculated rather precisely in the Standard Model (SM)[7] as $A_{f s}^{d}=(-4.1 \pm 0.6) \times 10^{-4}$ and $A_{f s}^{s}=(1.9 \pm 0.3) \times 10^{-5} . A_{f s}^{s}$ suffers from an additional CKM suppression factor $\lambda^{2}=0.22^{2}$ with respect to $A_{f_{s}}^{d}$. The leading-order contributions are given by so-called box diagrams, and New Physics contributions could enhance the flavour specific asymmetries to the percent level $[7,8]$.

\section{Measurement of $A_{f s}^{s}$}

A direct measurement of $A_{f s}$ requires flavour tagging at decay time, which is most efficiently done using semileptonic $B$-decays. Flavour tagging at production is very expensive in terms of statistics at a hadron collider. At LHCb, using same and opposite side tagging, it would reduce the statistical power by about a factor of 30 . A more efficient method is using untagged semileptonic asymmetries, either time dependent or time integrated:

$$
\begin{aligned}
\frac{R\left(B \rightarrow \mu^{+} X_{c} v_{\mu}\right)(t)-R\left(B \rightarrow \mu^{-} \bar{X}_{c} \bar{v}_{\mu}\right)(t)}{R\left(B \rightarrow \mu^{+} X_{c} v_{\mu}\right)(t)+R\left(B \rightarrow \mu^{-} \bar{X}_{c} \bar{v}_{\mu}\right)(t)} & =\frac{A_{f s}}{2}+\left(A_{P}-\frac{A_{f s}}{2}\right)\left(\frac{\cos \Delta m t}{\cosh \Delta \Gamma t / 2}\right) \\
\frac{N\left(B \rightarrow \mu^{+} X_{c} v_{\mu}\right)-N\left(B \rightarrow \mu^{-} \bar{X}_{c} \bar{v}_{\mu}\right)}{N\left(B \rightarrow \mu^{+} X_{c} v_{\mu}\right)+N\left(B \rightarrow \mu^{-} \bar{X}_{c} \bar{v}_{\mu}\right)} & =\frac{A_{f s}}{2}+\left(A_{P}-\frac{A_{f s}}{2}\right)\left(1+\left(\frac{\Delta m}{\bar{\Gamma}}\right)^{2}\right)^{-1}
\end{aligned}
$$

The price to pay is a factor 2 loss in sensitivity and a dependence on the $B$ production asymmetry $A_{P}=\frac{N\left(B_{t=0}\right)-N\left(\bar{B}_{t=0}\right)}{N\left(B_{t=0}\right)+N\left(\bar{B}_{t=0}\right)}=\mathscr{O}(1 \%)$. For the $B_{s}^{0}$ system, thanks to the fast oscillation rate, $\frac{\Delta m_{s}}{\bar{\Gamma}} \approx 26$, the correction due to the production asymmetry turns out to be negligible within the present statistical precision, $<4 \times 10^{-5}$, while for the $B^{0}$ system, a time dependent analysis is needed to determine the correction due to $A_{P}$. $\mathrm{LHCb}$ decided to measure first the flavour specific asymmetry in $B_{s}^{0}$ with a time-integrated method, and develop in parallel all tools for a time dependent analysis for the $B^{0}$ system. This method is then challenged by determining the mixing parameters in the $B^{0}$ and $B_{s}^{0}$ systems (see section 4).

For the measurement of $A_{f s}^{s}$, semileptonic decays $B_{s}^{0} \rightarrow D_{s}^{-} \mu^{+} X$ are used. The $D_{s}^{-}$meson is reconstructed through its decay to $\Phi(1020) \pi^{+}$and vertexed with the $\mu^{+}$to determine the $B_{s}^{0}$ decay vertex. The selection is based on particle identification, large transverse momentum, good vertexing and significant separation from the $p p$ collision point. The raw signal yields for both magnet polarities and both $D_{s}^{+}$charges are obtained from a fit of a double Gaussian for the signal and a second-order Chebyshev polynomial for the background (see Fig.1). In total, we have about 185000 events to play with.

As for all flavour specific asymmetries, the measurement is affected by detector induced charge asymmetries, either due to differences in hadronic cross sections, or due to left/right asymmetries 

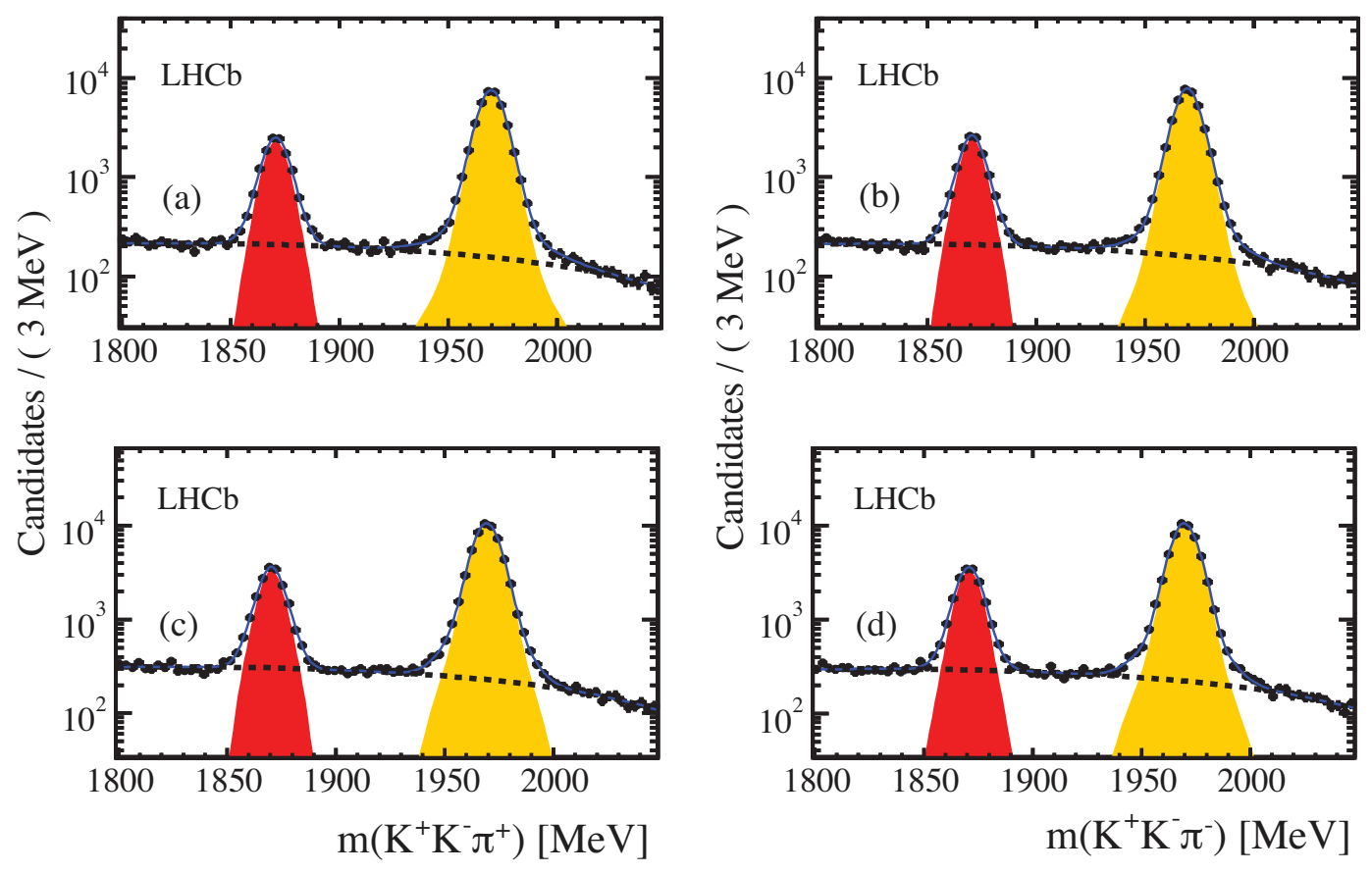

Figure 1: Invariant mass distributions for: (a) $K^{+} K^{-} \pi^{+}$and (b) $K^{+} K^{-} \pi^{-}$candidates for magnet up, (c) $K^{+} K^{-} \pi^{+}$and (d) $K^{+} K^{-} \pi^{-}$candidates for magnet down with $K^{+} K^{-}$invariant mass within $\pm 20 \mathrm{MeV}$ of the $\phi$ meson mass. The $D_{s}^{+}$[yellow (grey) shaded area] and $D^{+}$[red (dark) shaded area] signal shapes are described in the text.

of the LHCb detector together with the dipole field. The latter is by frequent change of polarities. However, this analysis is performed for both magnet polarities independently and both effects are controlled by using calibration channels.

The event yields are first corrected for muon related asymmetries caused by particle identification and the trigger selection by using high-statistics calibration channels of $J / \psi \rightarrow \mu^{+} \mu^{-}$. One sample is selected by requiring that events are triggered by a hadronic $B$ decay not including a $J / \psi$ in the final state, and a second sample by requiring that one of the muons of the $J / \psi$ decay fired the trigger. Using a tag and probe method, muon trigger and particle identification efficiencies, $\varepsilon\left(\mu^{-}\right)$and $\varepsilon\left(\mu^{+}\right)$, are determined in kinematic bins of the muon. Two different kinematic binning schemes are used, binning in $p_{x}$ and $p_{y}, p_{x}$ and $p_{y}$ being the Cartesian components of the muon momentum in the directions perpendicular to the beam axis, and binning in $p_{\mathrm{T}}$ and azimuthal angle $\phi$. Consistent results are obtained with both samples and the two kinematic binning schemes. For the final result, the average of all four combinations is used. In a second step, asymmetries caused by the track reconstruction are corrected for by using a sample of partially reconstructed charm decays. With this method [9], the tracking efficiency ratio of $\pi^{+}$over $\pi^{-}$can be determined as function of momentum with high precision. Since the interaction cross-section of $\pi^{+}$and $\pi^{-}$on isoscalar targets are equal at LHCb energies, the efficiency ratio can also be applied to muons, and we get for the combined tracking asymmetry of $\mu^{+} \pi^{-}$vs. $\mu^{-} \pi^{+}$a value of $A_{\text {track }}=(0.01 \pm 0.13) \%$. If the two kaon final state would be purely originating from the decay of the $\Phi$ resonance, no charge 
asymmetry due to kaons would appear. However, due to a small S-wave contribution, the $\mathrm{K}^{+}$ and $K^{-}$momenta are slightly different, which together with a kaon charge asymmetry determined from a combination of $D^{+}$meson decays to $K^{-} \pi^{+} \pi^{+}$and $K_{\mathrm{S}}^{0} \pi^{+}$, results in a small correction of $(0.012 \pm 0.004) \%$. Putting all together, we obtain:

$$
\begin{aligned}
A_{f s}^{s} & =2\left(\frac{N\left(D_{s}^{-} \mu^{+}\right)-N\left(D_{s}^{+} \mu^{-}\right) \times \frac{\varepsilon\left(\mu^{+}\right)}{\varepsilon\left(\mu^{-}\right)}}{N\left(D_{s}^{-} \mu^{+}\right)+N\left(D_{s}^{+} \mu^{-}\right) \times \frac{\varepsilon\left(\mu^{+}\right)}{\varepsilon\left(\mu^{-}\right)}}-A_{\text {track }}-A_{\mathrm{bkg}}\right) \\
& =(-0.06 \pm 0.50 \pm 0.36) \%
\end{aligned}
$$

The result is compatible with the Standard Model expectation and the most precise determination to date.

\section{Measurement of $B^{0}$ and $B_{s}^{0}$ mixing}

The measurement of the mixing asymmetries and the determination of the oscillation frequencies using semileptonic $B^{0}$ and $B_{s}^{0}$ decays alone is the first step towards a time-dependent analysis for measuring $A_{f s}^{d}$. The mixing asymmetry $A_{\Delta m}(t)$ is the asymmetry between the observed rate of unmixed events, same $b$ flavour at production and decay time, and mixed events, different $b$ flavour at production and decay time:

$$
A_{\Delta m}(t)=\frac{(R(\bar{B} \rightarrow \bar{B})(t)+R(B \rightarrow B)(t))-(R(\bar{B} \rightarrow B)(t)+R(B \rightarrow \bar{B})(t))}{(R(\bar{B} \rightarrow \bar{B})(t)+R(B \rightarrow B)(t))+(R(\bar{B} \rightarrow B)(t)+R(B \rightarrow \bar{B})(t))}=\frac{\cos \Delta m t}{\cosh \frac{\Delta \Gamma}{2}}
$$

The following semileptonic decay channels are used: $B_{d, s}^{0} \rightarrow D_{(s)}^{-}\left(\rightarrow K^{+} K^{-} \pi^{-}\right) \mu^{+} v_{\mu}$ without any requirement on the invariant mass of the two kaons. The flavour at production is tagged with the standard LHCb procedure of using opposite side and same-side tagging algorithms exploring the correlated production of flavour in strong interactions [10, 11, 12].

The determination of the $B$-hadron decay time is complicated by missing particles, mainly by the missing neutrino. A k-factor approach is being used, where the k-factor corrects in average for the missing momentum and is obtained from MC studies tuned to describe real data. The average k-factor is parameterized with a 4th-order polynomial as function of the normalized $D_{(s)}^{-} \mu^{-}$ invariant mass. The larger the $D_{(s)}^{-} \mu^{+}$invariant mass is, the smaller the spread of the k-factor, since less phasespace is left for the missing particle. The k-factor spread dominates the lifetime resolution which results in a decay time dependent decay time resolution. The mixing asymmetry gets additionally distorted by the resolution convoluted with the decay time acceptance, by background and by the mistag fraction. All these contributions are taken care of in a binned multidimensional log-likelihood fit applied to the same and opposite sign decay rates [4]. Fig.2 shows the mixing asymmetries and the projection of the fitted PDF, around the $D_{s}^{+}$mass peak, showing $B_{s}^{0}$ oscillations, and around the $D^{+}$mass peak showing $B^{0}$ oscillations. The total p-value of the fit is $19.6 \%$, the no mixing hypothesis is rejected by $5.8 \sigma$ for the $B_{s}^{0}$ and by $13 \sigma$ for the $B^{0}$ system. The extracted $B^{0}$ and $B_{s}^{0}$ mixing frequencies are $\Delta m_{d}=\left(0.503 \pm 0.011_{\text {stat. }} \pm 0.013_{\text {sys. }}\right) \mathrm{ps}^{-1}$ and $\Delta m_{s}=\left(17.93 \pm 0.22_{\text {stat. }} \pm 0.15_{\text {sys. }}\right) \mathrm{ps}^{-1}$, respectively. 

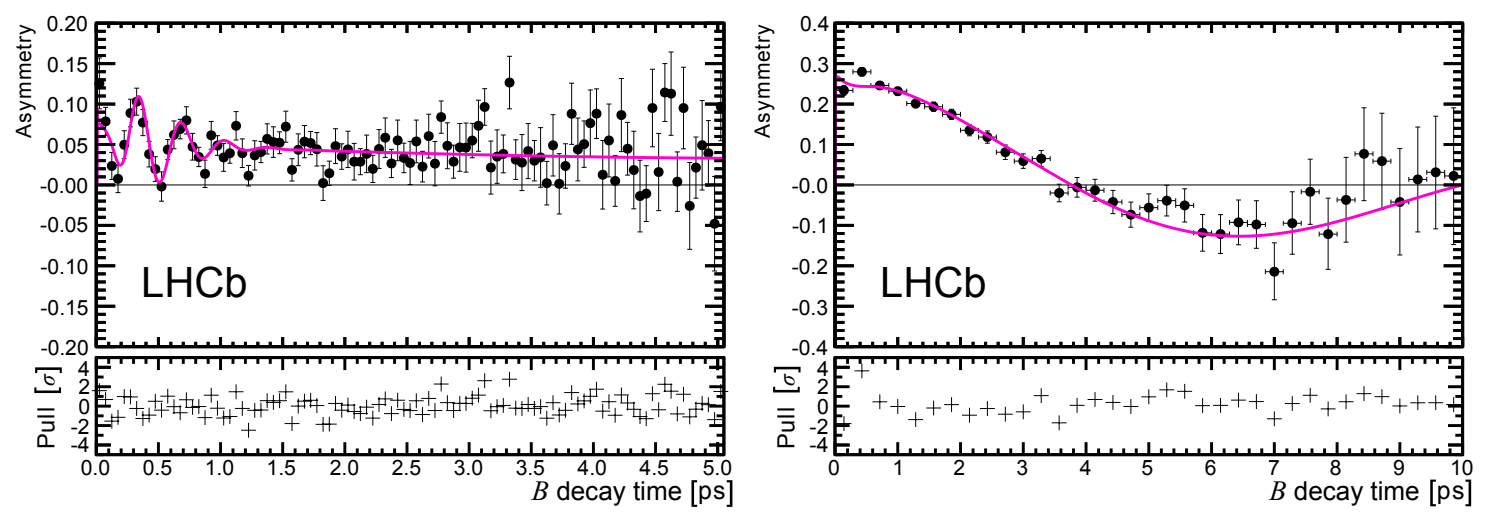

Figure 2: Tagged (mixing) asymmetry, $A_{\Delta m}$, as a function of $B$ decay time. The left plot shows the asymmetry for events for a region of $\pm 20 \mathrm{MeV} c^{-2}$ around the $D_{s}^{+}$mass peak, and the right plot shows the corresponding asymmetry around the $D$ mass peak. The black points show the data and the curves are projections of the fitted PDF.

\section{Conclusions}

LHCb measured with $1 \mathrm{fb}^{-1}$ of data the flavour specific asymmetry $A_{f s}^{s}$ with high precision. The result is consistent with the SM expectation. A first measurement of $\Delta m_{s}$ with semileptonic decays only has been performed, proofing that $\mathrm{LHCb}$ is on the right track to measure $A_{f s}^{d}$. Using Cabibbo favoured $D$ decays, a statistical precision of $\sim 0.2 \%$ could be expected with the recorded data of $3 \mathrm{fb}^{-1}$. A significant increase of statistics in the coming years is expected. Starting in 2015 , LHC will run at $\approx 13 \mathrm{TeV}$, almost doubling the $b \bar{b}$-cross section. With the LHCb upgrade after 2018, we expect to collect $50 \mathrm{fb}^{-1}$, which would correspond to more than hundred times the statistics of the analyses presented in this talk.

\section{References}

[1] LHCb collaboration, A. A. Alves Jr. et al., JINST 3 (2008) S08005.

[2] R. Aaij et al., JINST 8 (2013) P04022, arXiv: 1211. 3055.

[3] LHCb collaboration, R. Aaij et al., arXiv:1308.1048, submitted to Phys. Lett. B.

[4] LHCb collaboration, R. Aaij et al., arXiv:1308.1302, submitted to Eur. Phys. J. C.

[5] P. K. Kabir, Phys. Rev. D2 (1970) 540.

[6] CPLEAR collaboration, A. A. et al., Phys. Lett. B444 (1998) 52.

[7] A. Lenz, arXiv:1205.1444.

[8] C. Bobeth and U. Haisch, arXiv:1109.1826.

[9] LHCb collaboration, R. Aaij et al., Phys. Lett. B713 (2012) 186, arXiv: 1205 . 0897.

[10] M. Grabalosa, CERN-THESIS 075 (2012).

[11] LHCb collaboration, R. Aaij et al., Eur. Phys. J. C72 (2012) 2022, arXiv: 1202.4979.

[12] LHCb collaboration, R. Aaij et al., New J. Phys. 15 (2013) 053021, arXiv: 1304.4741. 